\title{
Usefulness of Hemodynamic Sensors for Physiologic Cardiac Pacing in Heart Failure Patients
}

\author{
Eraldo Occhetta, Miriam Bortnik, and Paolo Marino \\ Dipartimento Cardiologico, AOU Maggiore della Carità, Corso Mazzini 18, 28100 Novara, Italy \\ Correspondence should be addressed to Eraldo Occhetta, occhetta@r-j.it
}

Received 12 August 2010; Accepted 10 January 2011

Academic Editor: Georgios Giannakoulas

Copyright ( $) 2011$ Eraldo Occhetta et al. This is an open access article distributed under the Creative Commons Attribution License, which permits unrestricted use, distribution, and reproduction in any medium, provided the original work is properly cited.

\begin{abstract}
The rate adaptive sensors applied to cardiac pacing should respond as promptly as the normal sinus node with an highly specific and sensitive detection of the need of increasing heart rate. Sensors operating alone may not provide optimal heart responsiveness: central venous $\mathrm{pH}$ sensing, variations in the oxygen content of mixed venous blood, QT interval, breathing rate and pulmonary minute ventilation monitored by thoracic impedance variations, activity sensors. Using sensors that have different attributes but that work in a complementary manners offers distinct advantages. However, complicated sensors interactions may occur. Hemodynamic sensors detect changes in the hemodynamic performances of the heart, which partially depends on the autonomic nervous system-induced inotropic regulation of myocardial fibers. Specific hemodynamic sensors have been designed to measure different expression of the cardiac contraction strength: Peak Endocardial Acceleration (PEA), Closed Loop Stimulation (CLS) and TransValvular Impedance (TVI), guided by intraventricular impedance variations. Rate-responsive pacing is just one of the potential applications of hemodynamic sensors in implantable pacemakers. Other issues discussed in the paper include: hemodynamic monitoring for the optimal programmation and follow up of patients with cardiac resynchronization therapy; hemodynamic deterioration impact of tachyarrhythmias; hemodynamic upper rate limit control; monitoring and prevention of vasovagal malignant syncopes.
\end{abstract}

\section{Introduction}

The first implantable cardiac pacemakers were designed to pace at a fixed rate without any attempt to mimic heart rate changes. Since there, technologically advanced pacemakers were introduced that adjusted the pacing rate by sensing the patient's intrinsic atrial activity and regulating the ventricular pacing rate accordingly.

Successively, by the mid-1980s, artificial sensors were introduced in order to overcome problems with chronotropic incompetence in the pacemaker-recipient population. The prevalence of chronotropic incompetence is estimated to be present in $20 \%$ to $58 \%$ of pacemaker recipients $[1,2]$. In these patients, rate responsive pacing has been demonstrated to improve cardiac output and exercise tolerance when compared with fixed rate pacing [3]; many older studies have shown that exercise capacity, stroke volume changes with exercise, and maximum oxygen consumption depend primarily on heart rate change in most subjects [4-7].
Aim of the present paper is to address the different hemodynamic sensors currently implemented in rate responsive pacemakers.

\section{Rate Responsiveness Traditional Sensors}

Ideally, the rate-adaptive sensors should be capable of mimicking the sinoatrial's node ability to provide an appropriate heart rate response to the physiological and psychological stress and rate decay during recovery after exercise should match metabolic needs [8].

Many different types of rate-modulated cardiac pacemakers have been investigated.

In the mid-1970s, Cammilli et al. [9] provided the first implantable device using central venous $\mathrm{pH}$ sensing; the concept was that the decrease of central venous $\mathrm{pH}$ associated with exercise would increase pacemaker rate; unfortunately this sensor was not found to be stable in the long-term, and it was abandoned. 
Variations in the oxygen content of mixed venous blood, measured as the right ventricular oxygen saturation, have been successively evaluated as indicators for controlling the pacing rate [10]. The main technical problem involved in using this biosensor was that the signal received from the oxygen sensor showed dynamic fluctuations that depend on numerous factors: changing conditions of light reflection around the electrode, movements of erythrocytes with different oxygen saturation levels, incomplete mixing of blood, position of the oxygen sensor within the right ventricular cavity, and the direction of the light beam emitted [11].

The QT interval, which is the interval between the pacing stimulus to the peak of the $\mathrm{T}$ wave, is another parameter that has been investigated for controlling the pacing rate $[12,13]$. This interval is modified by changes in circulating or locally released cathecholamines, constituting a physiological indicator for adapting the pacing rate in response to increased sympathetic activity induced by exercise or emotion. However, the QT interval may be unreliable in T wave undersensing and is affected by drugs and electrolyte alterations; besides, as it requires ventricular pacing, it cannot be used in AAIR mode [8].

Respiration has been used as a physiological sensor to restore physiological rate control of the heart with a respiratory-dependent pacemaker [14]. Sensing of breathing rate and tidal volume was monitored by impedance variations initially detected between the pacemaker casing and an auxiliary lead implanted subcutaneously. The use of the respiratory rate as a pacing sensor was demonstrated to significantly improve exercise tolerance compared to fixed rate pacing [15]. A successful approach calculated minute ventilation by impedance measurements made between a bipolar pacemaker lead and the pulse generator case. This respiratory pacemaker changes the cardiac stimulation rate in response both to the rate of respiration and to the tidal volume, both of which make up minute ventilation, a value that has been demonstrated to correlate well with exercise [16]. Limitations of this sensor include lower reliability in subjects with obstructive pulmonary disease, false positive reaction in hyperventilation, or interference with posture [11].

Activity sensors are the older, and by far, the most popular and more widely used. Activity may be acknowledged either by a piezoelectric crystal which recognizes body movements or by an accelerometer that identifies the postural changes and the body movements related to physical activity [8].The main limitation of this kind of sensor is that the rate of adaptation may not be proportional to the metabolic demand. For example, activity sensors may underrespond to activities in which there is minimal thoracic movement such as smooth callisthenics movements, treadmill gradient change, and cycle ergometer exercise. These sensors may also yield an excessive heart rate response to tempo changes, staircase decent, and upper arm activity [11].

As shown before, sensors operating alone may not provide optimal heart rate responsiveness. Consequently, sensors that have different attributes but work in a complementary manner offers advantages [17]. However, complicated sensor interactions may occur.
The most frequent association includes the combination of an activity sensor which allows a rapid response during exercise and a metabolic sensor such as minute ventilation or QT interval which provides an increasing pacing rate during mental stress.

The LIFE study [18], which compared the relative benefit of a blended sensor (accelerometer and minute ventilation) versus single sensor (accelerometer), has demonstrated that the use of dual-sensor pacemaker restored chronotropic response and allowed a significant improvement of exercise capacity when compared with the use of only the accelerometer sensor. This seems particularly true in patients with marked chronotropic incompetence [19].

Pacemaker sensor features, alone or in combination, are frequently used in default setting; the role of individual optimization of the actual sophisticated sensors in improving quality of life and exercise capacity requires further investigation. A recent study has demonstrated that after 1 month of individual optimization of rate response pacemakers, exercise capacity was improved and maximum heart rate increased, although quality of life remained unchanged [20]. Besides, The ADEPT study [21] which was a single-blind randomized controlled trial comparing dual-chamber with rate-modulated dual-chamber pacing in patients with predominant sinus node dysfunction, failed to demonstrate the effectiveness of rate modulation in improving the functional status or quality of life.

\section{Rate Responsiveness Haemodynamic Sensors}

Hemodynamic sensors detect changes in the hemodynamic performances of the heart, which partially depends on the autonomic nervous system-induced inotropic regulation of myocardial fibers. Under physiological conditions, inotropic and chronotropic properties are controlled in order to synergistically modulate the cardiac output according to the metabolic demand. Once the required hemodynamic compensation is achieved, the system is downregulated. Thanks to this intrinsic negative feedback, in the case of chronotropic incompetence, assessment of cardiac contractility would allow timely rate adaptation with reduced risk of overpacing.

Specific haemodynamic sensors have been designed to measure different expression of the cardiac contraction strength.

The main intracardiac sensors which today seem to better measure surrogate parameters of hemodynamics include Peak Endocardial Acceleration (PEA) (Sorin Group, Italy), ventricular impedance guiding a Closed Loop Stimulation (CLS) (Biotronik, Germany), and Transvalvular Impedance (TVI (Medico, Italy).

3.1. Peak Endocardial Acceleration (PEA) (Figure 1). Theoretically, the contractile state of the heart can be identified in terms of ventricular mechanics by the maximum velocity of shortening of unloaded myocardial contractile elements.

More than 20 years ago, an experimental rate responsive pacemaker based on the detection of the peak $\mathrm{dP} / \mathrm{dt}$ of the right ventricle to drive the pacing rate was investigated 


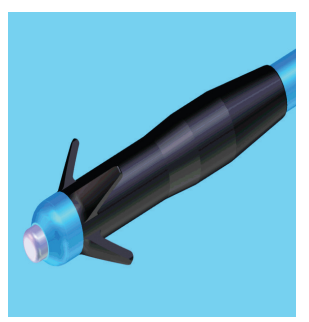

PEA-I component $=$ isovolumic contraction (first cardiac tone)

PEA-II component $=$ isovolumiv relaxation (second cardiac tone)
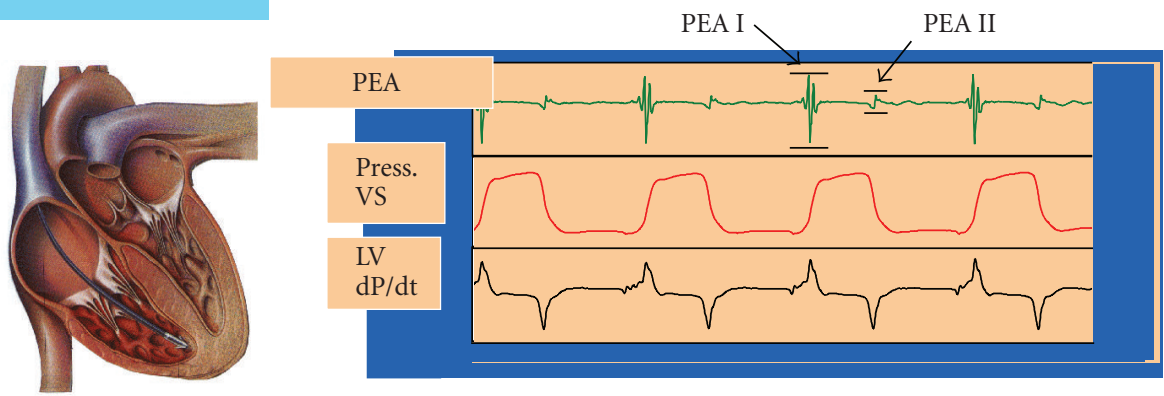

Figure 1: Peak Endocardial Acceleration (PEA) sensor (Sorin Group, Italy). An accelerometer mounted on the tip of a pacing lead placed in the right ventricle detects PEA-I, related to the isovolumic contraction (and the first cardiac tone) and PEA-II, related to the isovolumic relaxation (and the second cardiac tone).
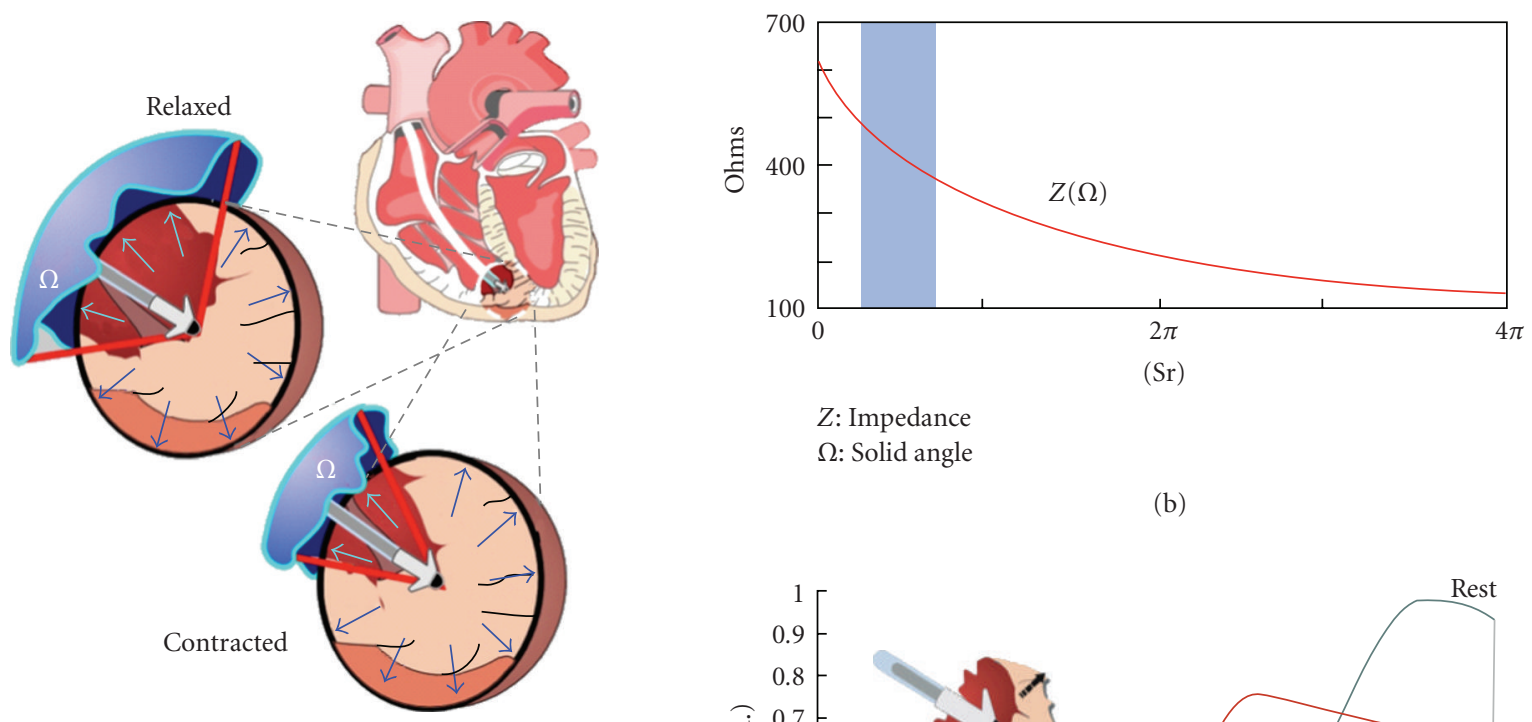

$(\mathrm{Sr})$

$Z$ : Impedance
$\Omega:$ Solid angle

(b)

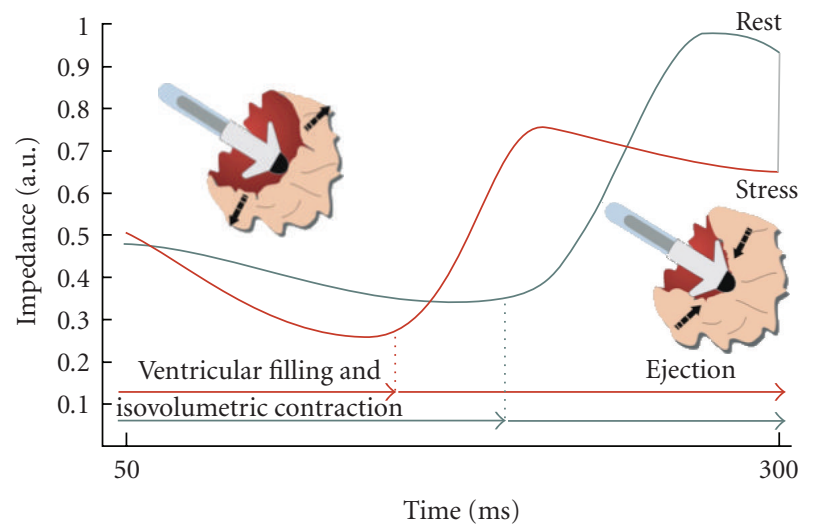

(c)

Figure 2: Closed Loop Stimulation (CLS) system (Biotronik, Germany). (a) Changes in intracardiac impedance are closely related to myocardium-blood ratio in the volume around the ventricular tip. (b) An increase in blood volume (and the consequent solid angle) produces a decrease in intracardiac impedance (Z). (c) An enhanced contractility induces an increase of impedance around the electrode tip (morphological modification of impedance curve moves it to the left). 
[22]; while the peak $\mathrm{dP} / \mathrm{dt}$ of the right ventricle has been demonstrated to reflect quite well the contractile property of the total heart, theoretically the main difficulties could be in long-term sensing of $\mathrm{dP} / \mathrm{dt}$, due to tissue encapsulation and ventricular muscle pressing on the membrane of the transducer.

The measurement of endocardial vibration, by means of an accelerometer in the right ventricle only during the isovolumetric phase, allows to assess a parameter of heart contractility: the systolic isovolumic peak acceleration, that was called "Peak Endocardial Acceleration (PEA)." PEA is recorded by an accelerometer mounted in the tip of a dedicated pacing lead which is sensitive to right ventricle wall vibrations generated by the mechanical activity of the heart; it was demonstrated to increase during adrenergic stimulation and to follow the changes in heart rate [23] Interestingly, even if the sensor is located in the right ventricle, the PEA amplitude is determined by the contraction strength in the left ventricle, where the systolic myocardial vibrations are generated. PEA dynamic monitoring has been demonstrated to provide fast pacing rate responses with long-term performance of sensor lead also in patients with heart failure and wide QRS complexes [24].

More recently, another application of the intracardiac accelerometer was reported. In addition to the first PEA (PEA I), recorded during the isovolumic systole, the sensor can detect a further signal, which is designated as PEA II. PEA II is recorded during the isovolumic relaxation and corresponds to the second cardiac sound. Experimental studies indicated that the PEA II amplitude reflects the rate of rise of the pressure gradient across the aortic valve at the time of valve closure, which depends on both the rate of ventricular pressure reduction (negative $\mathrm{dP} / \mathrm{dt}$ ) and the afterload [25], although its role in measuring diastolic function has not been confirmed yet.

Moreover, the PEA signal can be recorded at different locations in the heart; it has been recently demonstrated that the PEA signal measured in the atrium is reliable and proportional to the signal recorded in the ventricle [26].

The main disadvantage of the PEA recording comes for the need of a dedicated lead mounting the intracardiac accelerometer, which may limit the number of patients who could benefit from this sensor system.

3.2. Intracardiac Ventricular Impedance (Figure 2). An attractive method to obtain a physiological pacemaker system would be the integration of the pacing device into the natural cardiocirculatory system. This concept has been realized in the Closed Loop Stimulation (CLS) system, which converts information from the circulation applied to the right ventricle into a concordant heart rate [27]. Even under pathophysiological conditions, the dynamics of myocardial contraction still reflect the information coming from the circulation [28]. Inotropic regulation affects myocardial contractility, which consequently reflects information about the hemodynamic state and requirements. Based on that relationship, the CLS pacemakers from Biotronik (Biotronik $\mathrm{GmbH}$ et Co., Germany) detect changes in myocardial contraction dynamics through intracardiac measurement and transfer them into individual pacing rates. In these pacemakers, load-dependent changes in myocardial contractility reflect variations in the unipolar intracardiac impedance, measured between the ventricular electrode tip of a conventional bipolar lead and the pacemakers case. During myocardial contraction, the proportions of blood and myocardium vary in the close vicinity of the electrode tip. Since the specific resistance of blood differs significantly from that of the myocardium, the dynamics of the myocardial contraction can be well detected in a time-course impedance curve. Therefore, by monitoring the unipolar intracardiac impedance, changes in myocardial contractility can reliably be measured.

In a previous study [29], it has been demonstrated that CLS-driven pacemakers, in a population of patients with chronotropic incompetence, allowed overall cardiovascular responses comparable to those of healthy individuals throughout incremental exercise. Furthermore, the pacemaker provided appropriate rate response up to the anaerobic threshold. Additionally, the rate decay algorithm appeared to result in physiologically appropriate haemodynamics during the initial phase of recovery.

The role of CLS has also been well established in patients with refractory and recurrent vasovagal syncope even during a long-term follow-up [30-32]. The rationale for the effectiveness of CLS in these situations is that during a vasovagal syncope the diminished venous return stimulates a sympathetic compensatory tone that leads to a positive inotropic effect. Since ventricular filling is reduced, left ventricular systolic pressure may increase participating in a baroreceptor induction of bradycardia, thereby creating a paradoxical situation: increased inotropic effect associated with decreased chronotropic state. This anomalous situation inhibits sympathetic activity and promotes a reactive vagal effect that causes vasovagal syncope by increasing peripheral vasodilatation and reflex bradycardia. The CLS detection of the increased contractility in the first stage of vasovagal syncope could activate atrioventricular sequential pacing that may anticipate withdrawal of sympathetic tone and counterbalance the increase in vagal tone, preventing in this way arterial hypotension, bradycardia, and finally syncope.

The fourth generation of CLS devices, capable of operating on both sensed and paced ventricular beats, have overcome the major limitation of the previous systems which required permanent ventricular pacing.

3.3. Transvalvular Impedance (TVI) (Figures 3 and 4). Cardiac impedance could be applied to detect changes in end-diastolic ventricular volume as well as volume changes from diastolic to end-systolic conditions provided that the absolute impedance is recorded instead of just peak-topeak variation. This is possible if high-quality, stable, and noise-free impedance signals are derived. This goal has been achieved thanks to a recently developed recording method, whereby impedance is detected between the right atrium and the right ventricle, called transvalvular impedance (TVI). TVI is a regular periodic waveform with a minimum value during atrial systole (theoretically corresponding to the enddiastolic phase) and a maximum at the end of the QT period (theoretically corresponding to the end-systolic phase) [33]. 


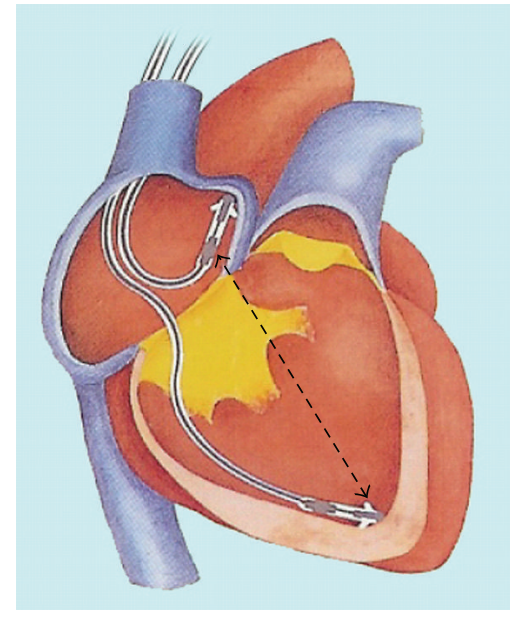

Aring-vtip TVI

(a)

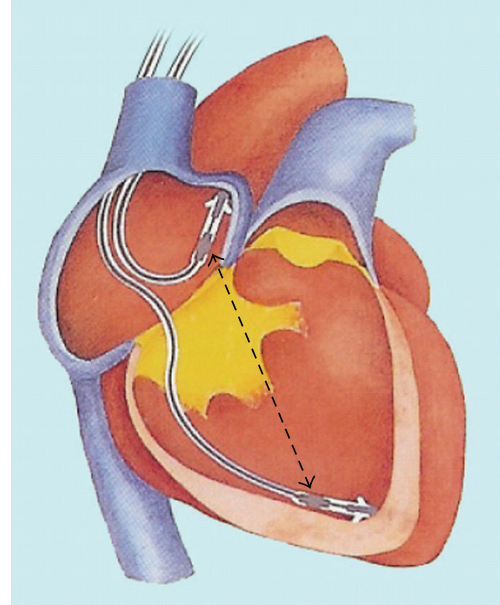

Aring-vring TVI

(b)

Figure 3: Trans Valvular Impedance (TVI) system (Medico, Italy). The impedance signal is derived between right atrium and the ventricle, by the tip (a) or the ring (b) electrodes of standard pacing leads.
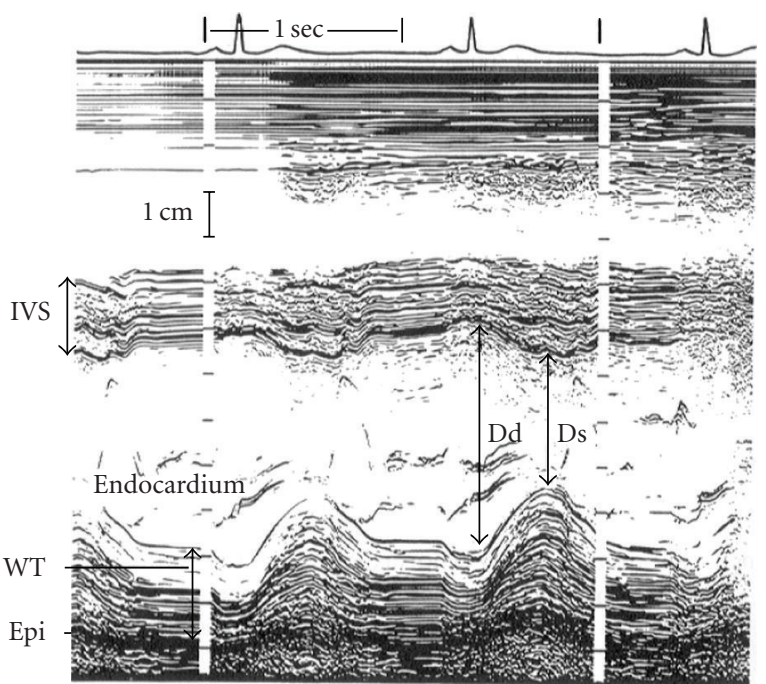

(a) Echocardiograph in an adult to show measurement of ventricular dimensions.
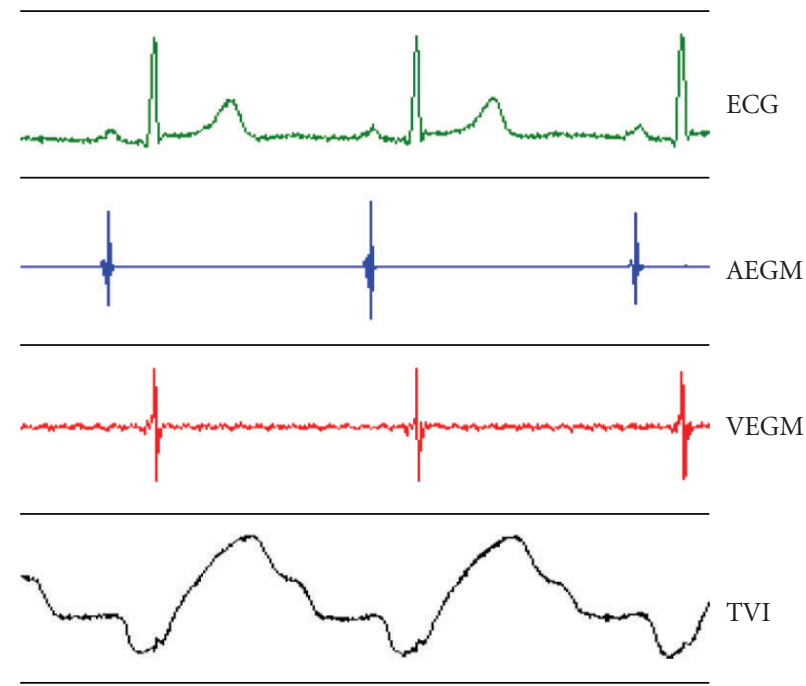

(b)

FIGURE 4: Trans Valvular Impedance (TVI) system (Medico, Italy). TVI waveform mirrors the time-course of ventricular volume along the cardiac cycle, detecting by M-mode Echo. TVI increases during ventricular systole and decreases during passive and active filling period.

The minimum TVI, which is recorded close to the maximum ventricular filling, is sensitive to all conditions known to modify the preload. The maximum TVI, which is recorded when the ventricular systole is completed, corresponds to the minimal ventricular volume (end-systolic volume) and is sensitive to changes in cardiac contractility. The relative variations, that could surrogate the end-diastolic and endsystolic volumes, left ventricular ejection fraction and stroke volume, define a cardiac inotropic index fully independent from preload effects, which is a direct expression of the autonomic nervous system regulation of the heart [34].
Even if TVI data are referred to the right ventricle, the essential haemodynamic information holds for the systemic circulation as well, since under steady conditions, the stroke volume is the same in the pulmonary artery and the aorta.

When inotropic index was used to drive a rate-responsive stimulator (SOPHÒS by Medico, Padova, Italy), the TVIindicated pacing rate proved a precise replication of the individual sinus rate in patients with physiological chronotropic competence [35].

The ability to discriminate the hemodynamic expression of cardiac contractility from the preload effects is 
an important advance in hemodynamic sensor technology, which is expected to improve pacing rate regulation in all circumstances entailing a modification in venous return. Furthermore, the prospect of obtaining diagnostic information on the trend of diastolic ventricular filling, as well as on the ejected blood volume, in changing daily-life conditions by an implanted device, makes TVI an appealing new tool in the medical care of pacemaker patients.

\section{Clinical Impact of Hemodynamic Sensors}

Rate-responsive pacing is just one of the many potential applications of haemodynamic sensors in implantable pacemakers, always blending with activity sensors. Specially in heart failure patients they could assure an optimal upper rate limit control, reducing deleterious inappropriate rate response induced by motion sensors [36].

If applied to a cardioverter defibrillator device (ICD), they could be used to discriminate the supraventricular and ventricular malignant tachyarrhythmias: its use could be a marker of hemodynamic deterioration during tachyarrhythmias and guide the ATP or shock therapy [37].

TVI has been proposed for beat-to-beat detection of mechanical ventricular activity and capture confirmation [38], with a haemodynamic alternative to the electrical autocapture control of the effective ventricular pacing.

Clinical trials support the use of these tools to recognize the prodromes of neurally mediated syncope and to trigger a protective increase in the cardiac pacing rate: this one could be possible as with PEA sensor [39] and with CLS pacing [30-32].

Closed loop stimulation and peak endocardial acceleration based algorithm has been recently demonstrated to be effective in atrioventricular delay optimal and dynamic programmation $[40,41]$. Besides, very recently, Becher et al. [42] have published the results of an animal study in which implant-based impedance showed a strong inverse correlation with changes of directly measured intrathoracic fluid accumulation.

In this setting, intrathoracic impedance monitoring, a hemodynamic sensor which is an index of changes of fluid status, even if not developed to drive heart rate, has been shown to reduce hospitalizations in heart failure patients [43]. Besides, when combined with other heart failure device diagnostic information such as long-lasting atrial fibrillation especially with rapid ventricular rate, low patient activity, high night heart rate, low heart rate variability could predict the clinical deterioration of ambulatory heart failure subjects, enhancing the ability to risk-stratify patients for subsequent heart failure events [44].

In cardiac resynchronization therapy, hemodynamic sensors could provide indications about the most appropriate left ventricular pacing site [45] or the best interventricular delay to apply [24-46].

Furthermore, hemodynamic sensors might play a role in the long-term monitoring of heart failure, helping the physician in the individual care of each patient. This last task would be quite appealing, especially if the diagnostic system gives information on the trend of the main hemodynamic variables such as the preload, the afterload, the stroke volume, and left ventricular ejection fraction [47].

\section{References}

[1] A. F. Rickards and R. M. Donaldson, "Rate-responsive pacing," Pacing and Clinical Electrophysiology, vol. 1, pp. 12-19, 1983.

[2] N. Gwinn, R. Leman, J. Kratz, J. K. White, M. R. Zile, and P. Gillette, "Chronotropic incompetence: a common and progressive finding in pacemaker patients," American Heart Journal, vol. 123, no. 5, pp. 1216-1219, 1992.

[3] S. K. Leung, C. P. Lau, and M. O. Tang, "Cardiac output is a sensitive indicator of difference in exercise performance between single and dual sensor pacemakers," Pacing and Clinical Electrophysiology, vol. 21, no. 1, pp. 35-41, 1998.

[4] I. Karloef, "Haemodynamic effect of atrial triggered versus fixed rate pacing at rest and during exercise in complete heart block," Acta Medica Scandinavica, vol. 197, no. 3, pp. 195-206, 1975.

[5] L. Fananapazir, D. H. Bennett, and P. Monks, "Atrial synchronized ventricular pacing: contribution of the chronotropic response to improved exercise performance," Pacing and Clinical Electrophysiology, vol. 6, no. 3, pp. 601-610, 1983.

[6] K. Ausubel, R. M. Steingart, M. Shimshi, P. Klementowicz, and S. Furman, "Maintenance of exercise stroke volume during ventricular versus atrial synchronous pacing: role of contractility," Circulation, vol. 72, no. 5, pp. 1037-1043, 1985.

[7] L. Ryden, O. Karlsson, and B. E. Kristensson, "The importance of different atrioventricular intervals for exercise capacity," Pacing and Clinical Electrophysiology, vol. 11, no. 7, pp. 10511062, 1988.

[8] S. Dell'Orto, P. Valli, and E. M. Greco, "Sensors for rate responsive pacing," Indian Pacing and Electrophysiology Journal, vol. 4, no. 3, pp. 137-145, 2004.

[9] L. Cammilli, L. Alcidi, G. Papeschi, V. Wiechmann, L. Padeletti, and G. Grassi, "Preliminary experience with the $\mathrm{pH}$ triggered pacemaker," Pacing and Clinical Electrophysiology, vol. 1, no. 4, pp. 448-457, 1978.

[10] A. Wirtzfeld, L. Goedel Meinen, T. Bock et al., "Central venous oxygen saturation for the control of automatic rate-responsive pacing," Pacing and Clinical Electrophysiology, vol. 5, no. 6, pp. 829-835, 1982.

[11] P. Rossi, "Rate-responsive pacing: biosensor reliability and physiological sensitivity," Pacing and Clinical Electrophysiology, vol. 10, no. 3, pp. 454-466, 1987.

[12] A. F. Rickards, R. M. Donaldson, and H. J. Thalen, "The use of QT interval to determine pacing rate: early clinical experience," Pacing and Clinical Electrophysiology, vol. 6, no. 2, pp. 346-354, 1983.

[13] R. M. Donaldson, K. Fox, and A. F. Rickards, "Initial experience with a physiological, rate responsive pacemaker," British Medical Journal, vol. 286, no. 6366, pp. 667-671, 1983.

[14] P. Rossi, M. D. Prando, A. Magnani, F. Aina, G. Rognoni, and E. Occhetta, "Physiological sensitivity of respiratorydependent cardiac pacing: four-year follow-up," Pacing and Clinical Electrophysiology, vol. 11, no. 9, pp. 1267-1278, 1988.

[15] P. Rossi, G. Rognoni, E. Occhetta et al., "Respiration-dependent ventricular pacing compared with fixed ventricular and atrial-ventricular synchronous pacing: aerobic and hemodynamic variables," Journal of the American College of Cardiology, vol. 6, no. 3, pp. 646-652, 1985. 
[16] F. Vai, J. L. Bonnet, P. Ritter, and G. Pioger, "Relationship between heart rate and minute ventilation, tidal volume and respiratory rate during brief and low level exercise," Pacing and Clinical Electrophysiology, vol. 11, no. 11, pp. 1860-1865, 1988.

[17] D. G. Benditt, M. Mianulli, K. Lurie, S. Sakaguchi, and S. Adler, "Multiple-sensor systems for physiologic cardiac pacing," Annals of Internal Medicine, vol. 121, no. 12, pp. 960968, 1994.

[18] J. Coman, R. Freedman, B. A. Koplan et al., "A blended sensor restores chronotropic response more favorably than an accelerometer alone in pacemaker patients: the LIFE study results," Pacing and Clinical Electrophysiology, vol. 31, no. 11, pp. 1433-1442, 2008.

[19] L. Padeletti, P. Pieragnoli, L. Di Biase et al., "Is a dual-sensor pacemaker appropriate in patients with sino-atrial disease? Results from the DUSISLOG study," Pacing and Clinical Electrophysiology, vol. 29, no. 1, pp. 34-40, 2006.

[20] A. Erol-Yilmaz, T. A. Schrama, J. S. Tanka, J. G. Tijssen, A. A. Wilde, and R. Tukkie, "Individual optimization of pacing sensors improves exercise capacity without influencing quality of life," Pacing and Clinical Electrophysiology, vol. 28, no. 1, pp. 17-24, 2005.

[21] G. A. Lamas, J. D. Knight, M. O. Sweeney et al., "Impact of rate-modulated pacing on quality of life and exercise capacityevidence from the advanced elements of pacing randomized controlled trial (ADEPT)," Heart Rhythm, vol. 4, no. 9, pp. 1125-1132, 2007.

[22] A. Sharma, R. Sutton, T. Bennet et al., "Physiologic pacing based on beat-to-beat measurement of right ventricular $\mathrm{dP} / \mathrm{dT}$ max: initially feasibility studies in man," Journal of the American College of Cardiology, vol. 7, p. 3A, 1986.

[23] E. Occhetta, A. Perucca, G. Rognoni et al., "Experience with a new myocardial acceleration sensor during dobutamine infusion and exercise test," European Journal of Cardiac Pacing and Electrophysiology, vol. 5, no. 4, pp. 204-209, 1995.

[24] P. Bordachar, S. Garrigue, S. Reuter et al., "Hemodynamic assessment of right, left, and biventricular pacing by peak endocardial acceleration and echocardiography in patients with end-stage heart failure," Pacing and Clinical Electrophysiology, vol. 23, no. 11, pp. 1726-1730, 2000.

[25] G. Plicchi, E. Marcelli, M. Parlapiano, and T. Bombardini, "PEA I and PEA II based implantable haemodynamic monitor: pre clinical studies in sheep," Europace, vol. 4, no. 1, pp. 49-54, 2002.

[26] L. Kubler, M. Burban, F. Anselme et al., "Hemodynamics monitoring in the atrium with a peak endocardial acceleration sensor: preliminary results from a pilot study," Europace, vol. 10, supplement 1, p. 82, 2008.

[27] M. Schaldach and H. Hutten, "Intracardiac impedance to determine sympathetic activity in rate responsive pacing," Pacing and Clinical Electrophysiology, vol. 15, no. 11, pp. 17781786, 1992.

[28] A. M. Pichlmaier, D. Braile, E. Ebner et al., "Autonomic nervous system controlled closed loop cardiac pacing," Pacing and Clinical Electrophysiology, vol. 15, no. 11, pp. 1787-1791, 1992.

[29] L. Cook, C. Tomczak, E. Busse, J. Tsang, W. Wojcik, and R. G. Haennel, "Impact of a right ventricular impedance sensor on the cardiovascular responses to exercise in pacemaker dependent patients," Indian Pacing and Electrophysiology Journal, vol. 5, no. 3, pp. 160-174, 2005.

[30] E. Occhetta, M. Bortnik, and C. Vassanelli, "The DDDR closed loop stimulation for the prevention of vasovagal syncope: results from the INVASY prospective feasibility registry," Europace, vol. 5, no. 2, pp. 153-162, 2003.
[31] E. Occhetta, M. Bortnik, R. Audoglio et al., "Closed loop stimulation in prevention of vasovagal syncope. Inotropy controlled pacing in vasovagal syncope (INVASY): a multicentre randomized, single blind, controlled study," Europace, vol. 6, no. 6, pp. 538-547, 2004.

[32] E. Occhetta, G. Dell'Era, M. Bortnik et al., "A long-term follow-up in a selected population implanted with DDDR closed loop stimulation (CLS) for vasovagal syncope prevention," Europace, vol. 10, supplement 1, p. 72, 2008.

[33] F. Di Gregorio, A. Morra, M. Finesso, and M. G. Bongiorni, "Transvalvular impedance (TVI) recording under electrical and pharmocological cardiac stimulation," Pacing and Clinical Electrophysiology, vol. 19, no. 11, pp. 1689-1693, 1996.

[34] F. Di Gregorio, A. Curnis, A. Pettini et al., "Trans-valvular impedance (TVI) in the haemodynamic regulation of cardiac pacing," in Cardiovascular Diseases 2002, P. Mitro, D. Pella, R. Rybar, and G. Valocik, Eds., pp. 53-57, Monduzzi, Bologna, Italy, 2002.

[35] G. Gasparini, A. Curnis, M. Gulizia et al., "Rate-responsive pacing regulated by cardiac haemodynamics," Europace, vol. 7, no. 3, pp. 234-241, 2005.

[36] G. Payne, J. Spinelli, C. J. Garratt, and J. D. Skehan, "The optimal pacing rate: an unpredictable parameter," Pacing and Clinical Electrophysiology, vol. 20, no. 4, pp. 866-873, 1997.

[37] R. Chirife, "Hemodynamic assessment with implantable pacemakers: how feasible and reliable is it?" in Cardiac Arrhythmias 2003, A. Raviele, Ed., pp. 705-712, Springer, Milan, Italy, 2003.

[38] M. G. Bongiorni, E. Soldati, G. Arena et al., "Transvalvular impedante: does it allow automatic capture detection?" in Cardiac Arrhythmias 2003, A. Raviele, Ed., pp. 733-739, Springer, Milan, Italy, 2003.

[39] J. C. Deharo, J. P. Peyre, P. H. Ritter, T. Chalvidan, L. Le Tallec, and P. Djiane, "Treatment of malignant primary vasodepressive neurocardiogenic syncope with a rate responsive pacemaker driven by heart contractility," Pacing and Clinical Electrophysiology, vol. 21, no. 12, pp. 2688-2690, 1998.

[40] L. Padeletti, M. C. Porciani, P. Ritter et al., "Atrioventricular interval optimization in the right atrial appendage and interatrial septum pacing: a comparison between echo and peak endocardial acceleration measurements," Pacing and Clinical Electrophysiology, vol. 23, no. 11, pp. 1618-1622, 2000.

[41] A. P. Ravazzi, P. Diotallevi, M. F. Provera et al., "AV delay optimization using ventricular impedante," Europace, vol. 2, supplement C, p. C26, 2001.

[42] J. Becher, S. G. Kaufmann, S. Paule et al., "Device-based impedance measurement is a useful and accurate tool for direct assessment of intrathoracic fluid accumulation in heart failure," Europace, vol. 12, no. 5, pp. 731-740, 2010.

[43] D. Catanzariti, M. Lunati, M. Landolina et al., "Monitoring intrathoracic impedance with an implantable defibrillator reduces hospitalizations in patients with heart failure," Pacing and Clinical Electrophysiology, vol. 32, no. 3, pp. 363-370, 2009.

[44] D. J. Whellan, K. T. Ousdigian, S. M. Al-Khatib et al., "Combined heart failure device diagnostics identify patients at higher risk of subsequent heart failure hospitalizations: results from PARTNERS HF (Program to Access and Review Trending Information and Evaluate Correlation to Symptoms in Patients With Heart Failure) study," Journal of the American College of Cardiology, vol. 55, no. 17, pp. 1803-1810, 2010.

[45] P. P. Delnoy, E. Marcelli, H. Oudeluttikhuis et al., "Validation of a peak endocardial acceleration-based algorithm to optimize cardiac resynchronization: early clinical results," Europace, vol. 10, no. 7, pp. 801-808, 2008. 
[46] M. Bocchiardo, D. Caponi, P. Di Donna et al., "Optimization of resynchronisation therapy by intracardiac ventricular impedance," in New Advances in Heart Failure and Atrial Fibrillation, M. Gulizia, Ed., pp. 411-415, Springer, Milan, Italy, 2003.

[47] E. Occhetta, A. Magnani, M. Bortnik et al., "Hemodynamic sensors: their impact in clinical practice," in Cardiac Arrhythmias 2003, A. Raviele, Ed., pp. 713-718, Springer, Milan, Italy, 2003. 


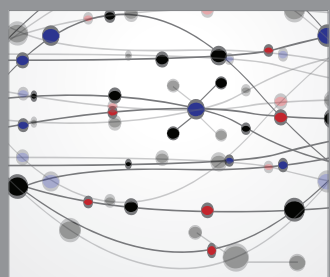

The Scientific World Journal
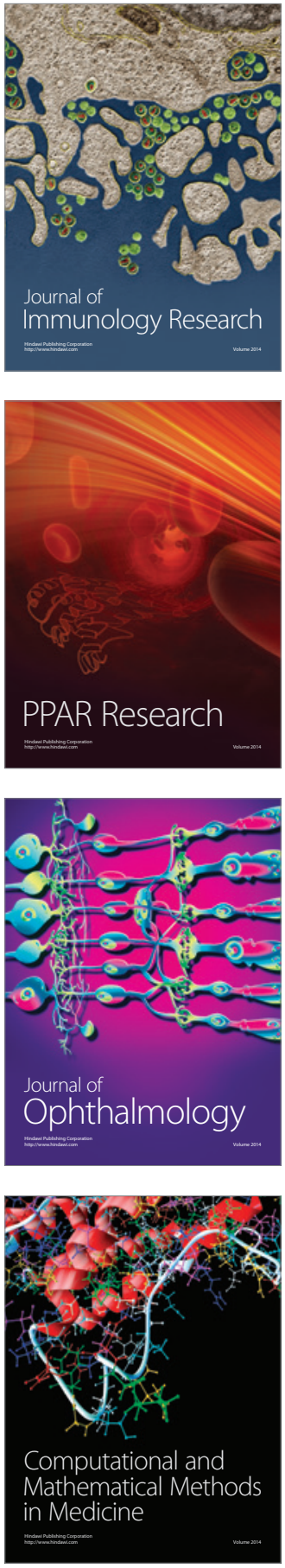

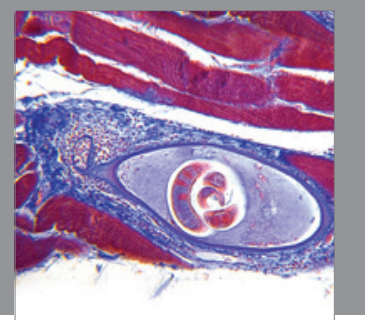

Gastroenterology

Research and Practice
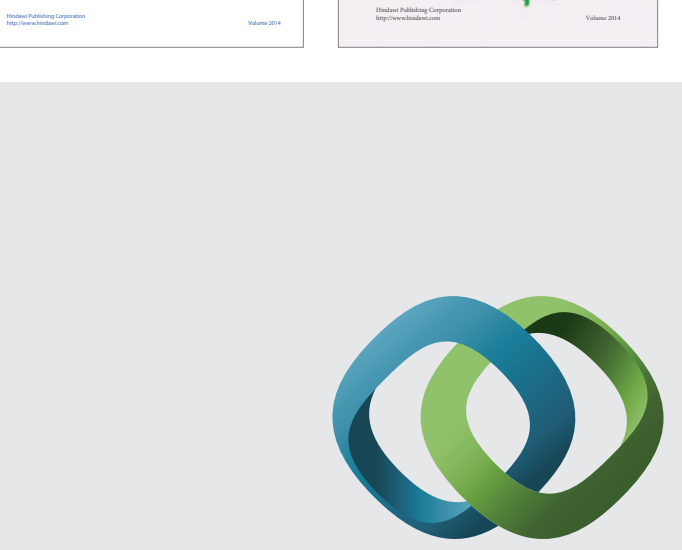

\section{Hindawi}

Submit your manuscripts at

http://www.hindawi.com
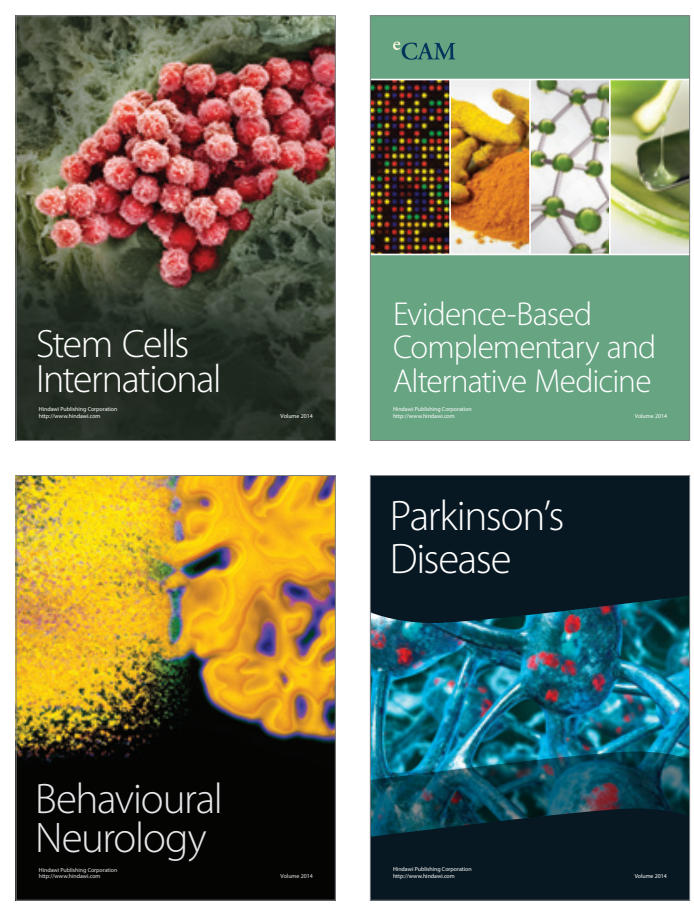

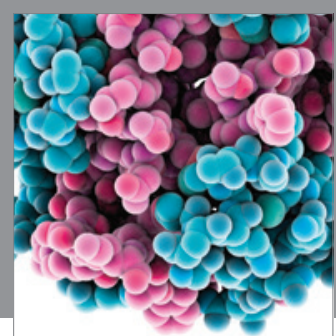

Journal of
Diabetes Research

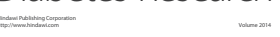

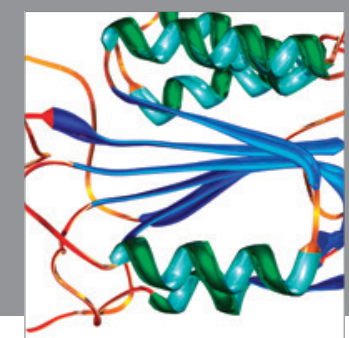

Disease Markers
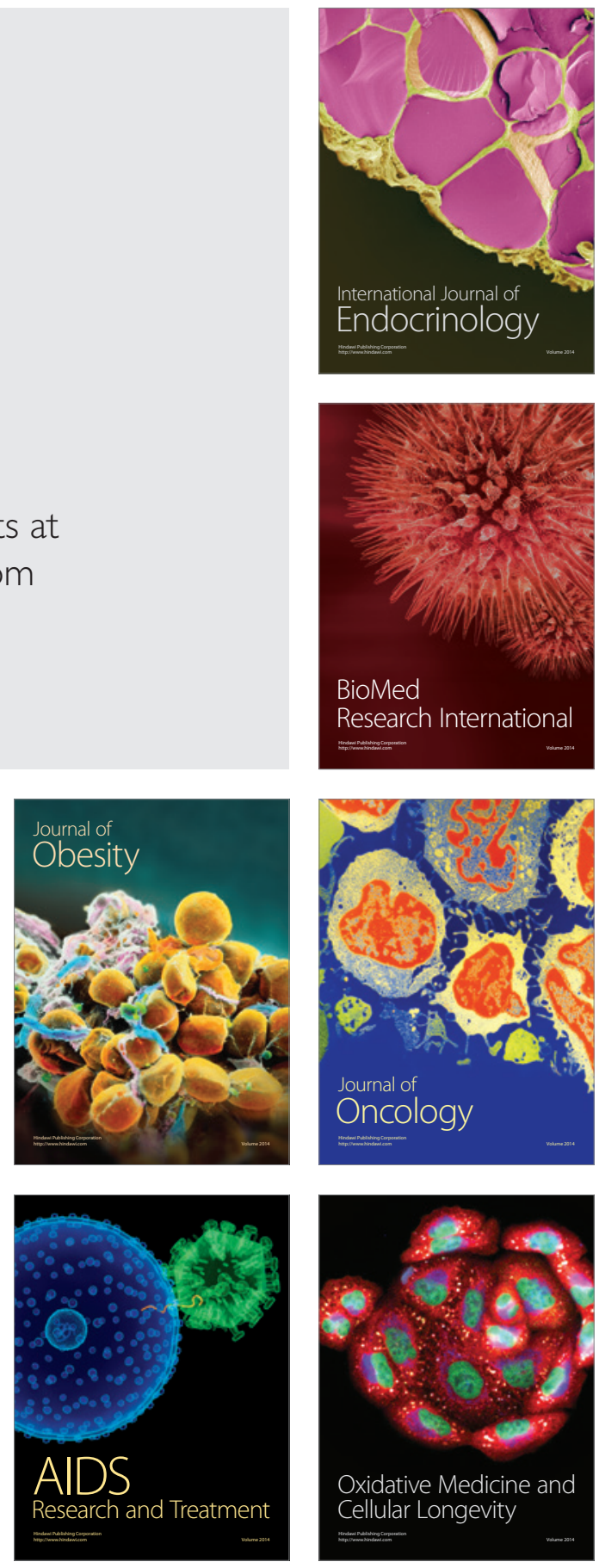